\title{
High Temperature and In Situ Study of SrO Surface Precipitation on Perovskite Ceramics
}

\author{
Mathew A. Niania ${ }^{1}$, Renaud Podor $^{2}$, T. Benjamin Britton ${ }^{1}$, Stephen J. Skinner ${ }^{1}$ and John A. Kilner ${ }^{1}$ \\ 1. Department of Materials, Imperial College London, London SW7 2AZ, UK. \\ 2. Institut de Chimie Séparative de Marcoule, UMR 5257 CEA/CNRS/UM/ENSCM, Site de Marcoule - \\ Bât. 426, BP 17171, 30207 Bagnols/Cèze cedex, France.
}

Solid oxide fuel cells (SOFC) convert gaseous fuels, e.g. $\mathrm{H}_{2}$, into electricity through an electrochemical process. Their conversion efficiencies are not limited by the Carnot cycle and pollution levels in the exhaust gas are significantly lower than that of traditional technologies. SOFC cathode materials require a very precise balance of material properties in order to function at operating temperatures $(\sim 600-$ $800^{\circ} \mathrm{C}$ ). A number of systems fulfil the requirements, but there are numerous challenges these materials face during manufacture and operation. Of particularly concern is the negative impact secondary phase formation at the surface has on the reduction of oxygen. In a large number of perovskite systems used for SOFC cathodes the A-site is occupied by lanthanum $\left(\mathrm{La}^{3+}\right)$ and it is often doped with strontium $\left(\mathrm{Sr}^{2+}\right)$ to introduce oxygen vacancies, which generates ionic conductivity, and electronic species leading to mixed conductivity, essential for operation as an SOFC cathode. The crystal chemistry of these perovskite structures can be described as an alternated stacking of $\mathrm{SrO}$ and $\mathrm{LaO}_{2}$ layers. It is believed that the charge difference between the lanthanum and strontium changes the state of the B transition metals (i.e. $\mathrm{B} 2+/ 3+$ ) to preserve charge neutrality and in turn creates dipole moments $\mathrm{SrO}$ and $\mathrm{LaO}_{2}$ layers. This creates an alternating electric field throughout the material, resulting in a large surface charge, which the system attempts to reduce by depleting the surface of La and segregating Sr [1].

Continuous surface precipitation was observed on polished $\mathrm{La}_{0.6} \mathrm{Sr}_{0.4} \mathrm{Co}_{0.2} \mathrm{Fe}_{0.8} \mathrm{O}_{3-\delta}$ (LSCF) ceramics using high temperature environmental scanning electron microscopy (HT-ESEM) up to $1000^{\circ} \mathrm{C}$. Several experiments were performed under different atmospheres: vacuum, $300 \mathrm{~Pa} \mathrm{O}_{2}, \mathrm{H}_{2} \mathrm{O}$ and air. A characteristic image series recorded under water vapor is reported on Figure 1. The surface precipitation phenomenon can be clearly observed. The composition of the precipitates determined from the X-ray maps recorded on samples cooled at room temperature is $\mathrm{SrO}$ (Fig. 2). The evolution of the surface occurs in three distinct stages: (1) rapid growth of precipitates on grain boundaries, at defect points as well as at the center of the grains, (2) agglomeration of the precipitates due to surface transport phenomena and (3) Continuous coarsening during the heat treatment. It is clear that at the beginning the precipitation process, the $\mathrm{SrO}$ precipitates appear to grow with crystallographic direction as, within each grain, they tend to orientate themselves in a similar direction. It is also clear that the initiation of the $\mathrm{SrO}$ precipitation and the density of the precipitates depend on the LSCF grain orientations. When comparing the EBSD maps with the HT-ESEM images (Fig. 3a), it is obvious that the precipitation process and grain growth are directly linked with the grain orientations and presence of twinning inside the grains (Fig. 3bcd). Twin planes exist in many grains. Average twin plane width measured across a particular grain (Fig. 3b) is very close to the average particle width (measured center to center), 0.6 and $0.5 \mu \mathrm{m}$ respectively. This result suggests that twin planes in this orientation are fast diffusion pathways. Other grains display homogenous precipitate growth across the grain surface and it is predicted that knowledge of the twin habit plane can be used to describe why these grain orientations display different behavior.

From these new data sets, a precise description of the surface precipitation has been proposed. 
References:

[1] WA Harrison, Phys Rev B 83 (2011), p. 155437.

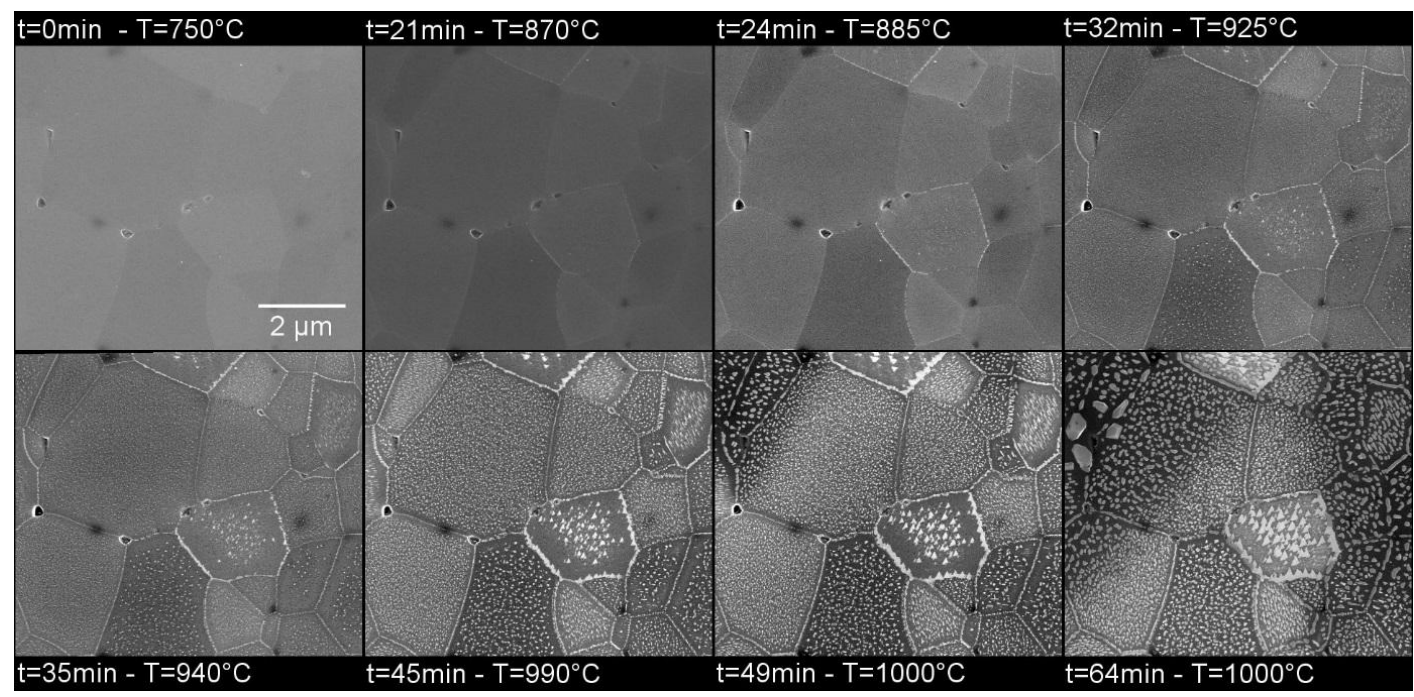

Figure 1. HT-ESEM micrographs of a $\mathrm{SrO}$ precipitation on a polished $\mathrm{La}_{0.6} \mathrm{Sr}_{0.4} \mathrm{Co}_{0.2} \mathrm{Fe}_{0.8} \mathrm{O}_{3-\delta}$ surface observed at high temperature in the ESEM under 300Pa of water vapour.

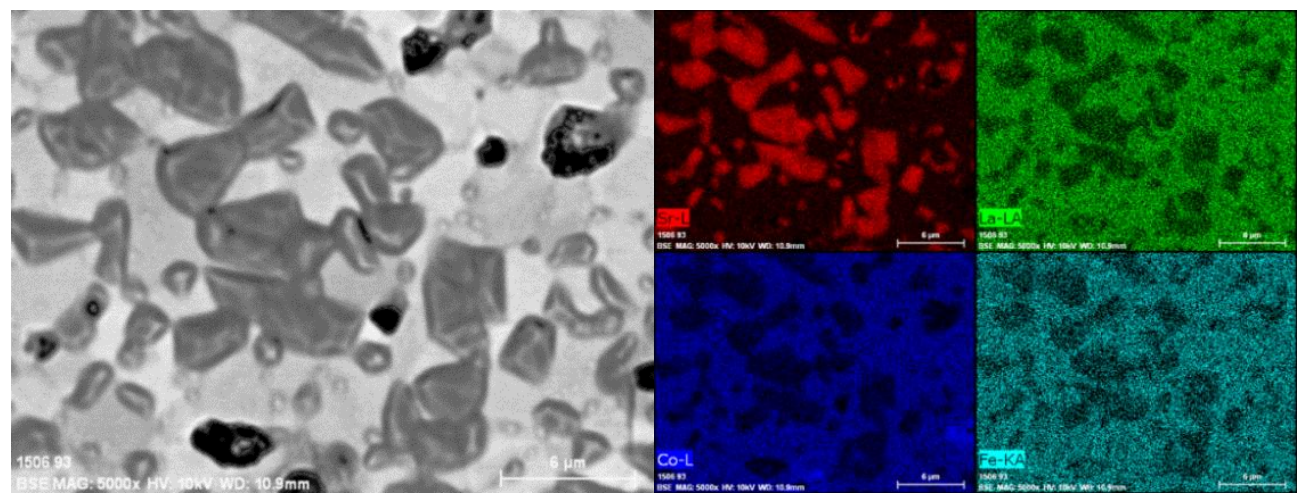

Figure 2. X-ray maps of $\mathrm{Sr}, \mathrm{La}, \mathrm{Co}$ and $\mathrm{Fe}$ recorded after the heat treatment of $\mathrm{La}_{0.6} \mathrm{Sr}_{0.4} \mathrm{Co}_{0.2} \mathrm{Fe}_{0.8} \mathrm{O}_{3-\delta}$ ceramic under $300 \mathrm{~Pa}$ of water vapour at $1000^{\circ} \mathrm{C}$.
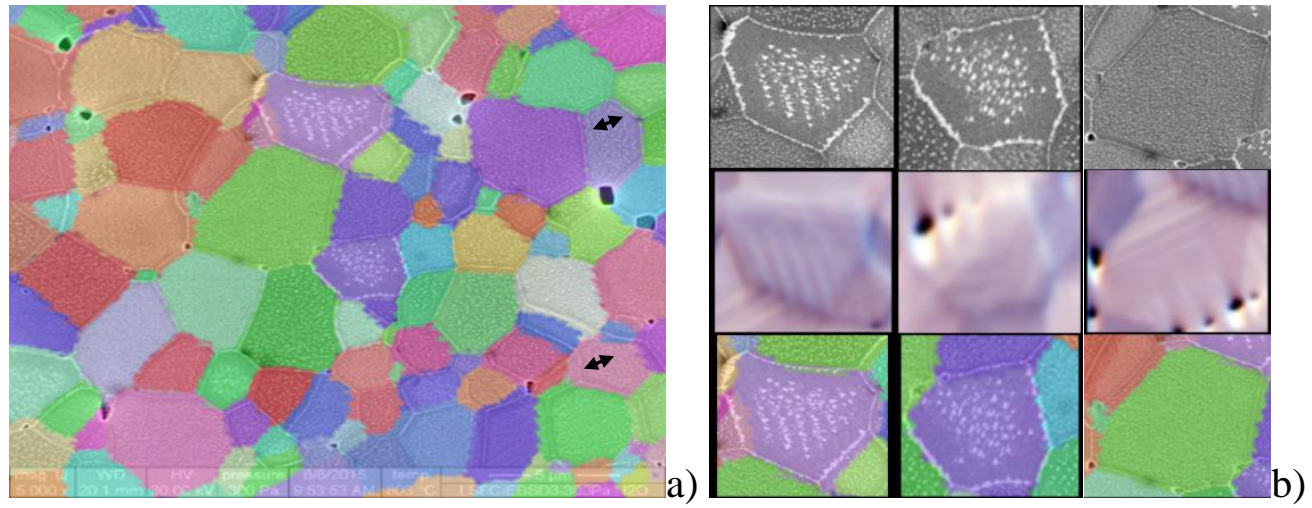

Figure 3. a) EBSD data matched with HT-ESEM data. b) Correlations between the HT-ESEM, argus and EBSD images determined for 3 specific grains. 